\title{
Oxygen doping of HTSC and resistive switching in HTSC-based heterostructures
}

\author{
Natalia A Tulina' ${ }^{1}$ Ivan Yu Borisenko², Andrey A Ivanov ${ }^{3 *}$, Andrey M Ionov and Ivan M Shmytko ${ }^{1}$
}

\begin{abstract}
The studies of the bipolar resistive switching effect in thin film heterojunctions $\left(\mathrm{YBa}_{2} \mathrm{Cu}_{3} \mathrm{O}_{7-\delta} / \mathrm{Ag}\right)$ and $\left(\mathrm{Nd}_{2-x} \mathrm{Ce}_{x} \mathrm{CuO}_{4-y} / \mathrm{Ag}\right)$ have exhibited the role of oxygen as a doping element in hole- and electron-doped HTSC compounds.
\end{abstract}

\section{Introduction}

Most HTSC materials are hole-doped compounds. $\mathrm{Nd}_{2-x} \mathrm{Ce}_{x} \mathrm{CuO}_{4-y}$ (NCCO), $\mathrm{Pr}_{2-x} \mathrm{Ce}_{x} \mathrm{CuO}_{4-y}$ (PCCO), as well as $\mathrm{Ba}_{1-x} K_{x} \mathrm{BiO}_{3-y}$ and $\mathrm{BaPb}_{1-x} \mathrm{Bi}_{x} \mathrm{O}_{3-y}$ are few representatives of electron-doped ones. The hole- and electron-doped compounds differ by their physical properties and their phase diagrams (Dagotto 1994). Nevertheless, they have a feature in common. It is strong dependence of the properties on oxygen stoichiometry, since oxygen plays significant role in formation of the basic (ground) state in these compounds. Influence of oxygen is well studiled for p-type HTSC, it is important as hole dopant. However, questions still remain concerning the role of oxygen in electron-doped HTSC cuprates (Orenstein and Wishwanath 2010). The studies of Hall effect (Jiang et al. 1994; Mao et al. 1995) in oxygenreduced NCCO and PCCO revealed presence of holelike states in these $n$-type materials. The question arises whether the presence of p-type carriers is crucial for superconductivity in HTSC.

The effect of bipolar resistive switching (BRS) observed in oxide-based heterostructures, including based on strongly correlated electron systems (SCES), such as HTSC (see, for instance, (Waser and Aono 2007; Tulina 2007; Sawa 2008; Kang et al. 2007; Yang et al. 2008; Inoue et al. 2008)), is sensitive to type of charge carriers in SCES. BRS is exhibited in SCES-normal metal heterocontacts at specific polarity of the electric field as a change of the phase composition of the SCES surface layer at the nanosize level. As a result,

\footnotetext{
*Correspondence: andrej.ivanov@gmail.com

3 National Research Nuclear University "MEPhl", Moscow, Russia

Full list of author information is available at the end of the article
}

the metastable high resistance (OFF) and low resistance $(\mathrm{ON})$ states of the heterocontact are realized leading to colossal electrical resistance (CER)(Tulina 2007). CER is the ratio of the resistance on OFF state to the resistance in $\mathrm{ON}$ state and characterizes the memory effect. $C E R=\Delta \mathrm{R} / \mathrm{R}=\left(\mathrm{R}_{O F F}(\mathrm{~V}=0)-R_{O N}(\mathrm{~V}=0)\right) / \mathrm{R}_{O N}(\mathrm{~V}=0)$. Intensive fundamental and application studies of this phenomenon are underway since BRS-based devices are regarded as a new generation of alternative nonvolatile memory (Meijer 2008). Besides, BRS effect allows to study the microscopic nature of SCES underlying such devices.

Recent studies of the BRS effect in various compounds revealed the essential role of the oxygen diffusion processes. Electric field of different polarity induces two resistive states of the degraded HTSC surface which is indicative of its separation into conducting and dielectric phases. The switching process gives rise to metastable states with different oxygen content in the interface area of the compound. It is this area that determines the resistive properties of the structure (Tulina et al. 2001; Tulina and Sirotkin 2004). Therefore, the BRS effect involves reproducible oxygen doping of a specific sample area. In (Tulina and Klinkova 2007) we observed BRS inversion in heterojunctions based on electron-doped $\mathrm{BaK}_{0,6} \mathrm{Bi}_{0,4} \mathrm{O}_{3-y}$ : the switching to the high resistance metastable phase takes place when the HTSC crystal has a negative potential with respect to the normal electrode. In this case the current field $(J=\sigma E)$ is directed to the surface in contrast to switching observed in hole-doped compounds.

\section{包 Springer}

(C) 2013 Tulina et al.; licensee Springer. This is an Open Access article distributed under the terms of the Creative Commons Attribution License (http://creativecommons.org/licenses/by/2.0), which permits unrestricted use, distribution, and reproduction in any medium, provided the original work is properly cited. 


\section{Experiment}

The aim of the work was to observe bipolar resistive switching effect in $\mathrm{YBa}_{2} \mathrm{Cu}_{3} \mathrm{O}_{7-\delta} / \mathrm{Ag}$ and $\mathrm{NCCO} / \mathrm{Ag}$ heterojunctions in order to trace the role of oxygen as doping element in p-type and n-type HTSC. Thin epitaxial films of $\mathrm{YBCO}(001)$ and $\mathrm{NCCO}(001)$ grown by pulse laser deposition were used to produce HTSC-based heterostructures. Figure 1 shows a heterocontact scheme. Silver counterelectrodes were deposited on the as-grown surface to form a HTSC film/interface/Ag structure. The upper silver electrode was deposited through a mask as point electrodes $1 \mathrm{~mm}$ in diameter. The electric supply leads were attached to the silver contacts with conductive paste or a mechanical contact in a micromanipulator. The $\mathrm{x}$-ray studies of the YBCO films has revealed that, besides the basic composition, sample contains surface regions with a smoothly decreasing oxygen content down to $\delta=1,0$ (Jorgensen et al. 1990).

Results of NCCO films structure study were reported elsewhere (Tulina et al. 2011). There were detected reflexes of $\mathrm{NCCO}$ and $\mathrm{Nd}_{0.5} \mathrm{Ce}_{0.5} \mathrm{O}_{1.75}(\mathrm{NCO})$ phase. Both phases were epitaxial and (001)-oriented. The obtained results are consistent with other evidences of two phases coexistence after oxygen reducing even in perfect single crystals (Mang et al. 2004). Along with the main phase, the second phase can affect the behavior of the interface of the studied heterojunctions and lead to changes in the type of conductivity. Photoemission studies provided support for the X-ray data. After the film surface was milled with nitrogen ions at depth of $50 \mathrm{~nm}$, it was shown using X-ray photoelectron spectroscopy (XPS) and ultraviolet photoelectron spectroscopy that the film surface was metallic and had an entire set of elements (Figure 1). We thus obtained heterojunctions of two types on NCCO films: (a) with a surface buffer layer formed by NCO (Figure 1a; see the XPS data above) and (b) without the NCO oxide layer, which was removed by ion milling to the main phase $\mathrm{NCCO}$ under photoemission control (Figure 1).

The value of the BRS effect was insignificant in the heterocontacts formed immediately after the ion milling of NCCO surface, the switching pattern corresponded to the electron-doped systems (see Figure 2). This fact can be understood considering weak influence of oxygen as a doping element on the properties of $\mathrm{NCCO}$ which has been already confirmed in some works (Higgins et al. 2006; Gauthier et al. 2007). Nonstoichiometric oxygen does not affect the number of carriers in NCCO and leads a) Ion milling at $10-15 \mathrm{~nm}$

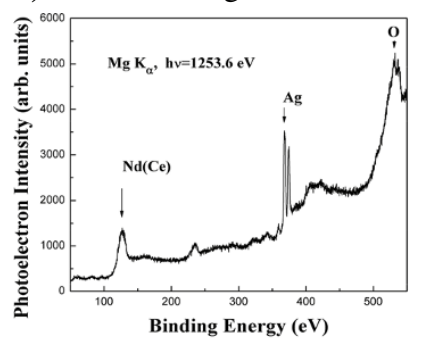

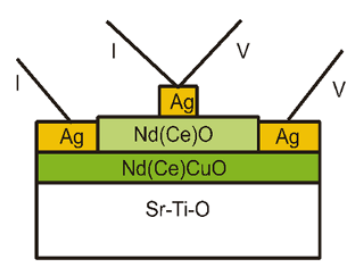

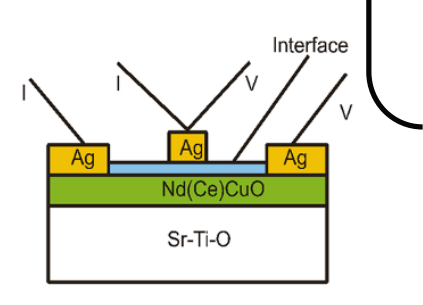

b)

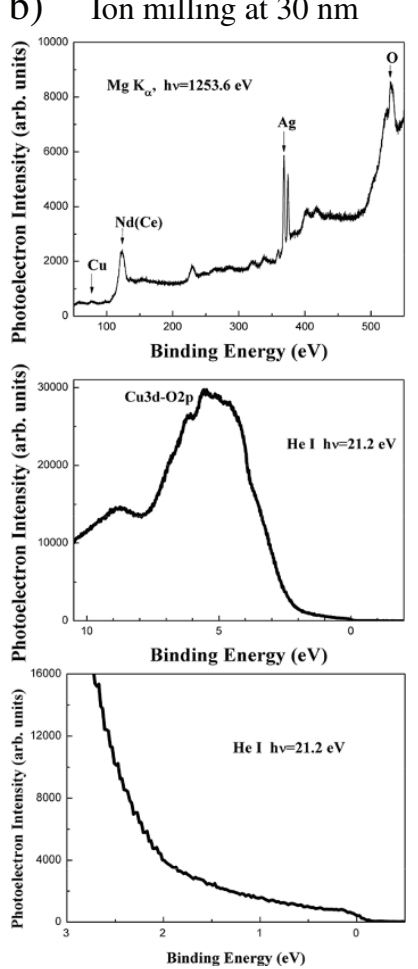

Figure $1 \mathrm{XPS}$ and UPS of the as grown and ion milled $\mathrm{Nd}_{1.75} \mathrm{Ce}_{\mathbf{0 . 1 5}} \mathrm{CuO} \mathrm{O}_{4-\boldsymbol{y}}$ films. (a) Initial stage of ion milling (up to 10-15 nm depth). XPS spectrum of the film surface (top), corresponding heterocontact scheme (bottom); (b) lon milling at $30 \mathrm{~nm}$ depth. XPS spectrum (top) and UPS spectra (middle and bottom) together with the heterocontact scheme. 


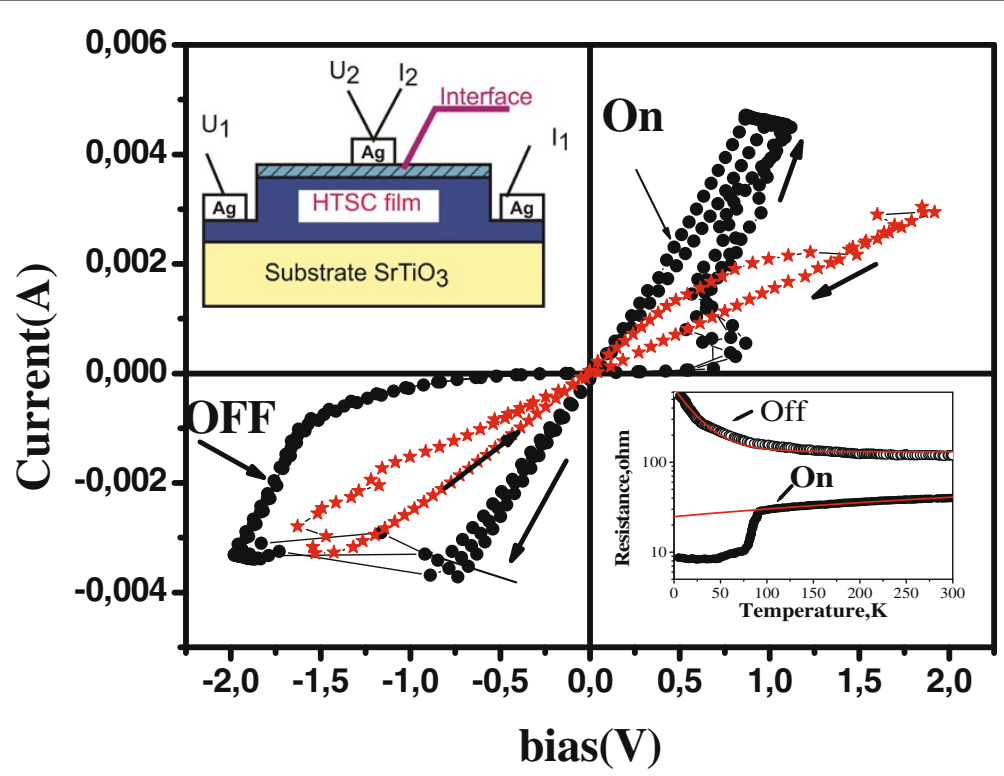

Figure 2 Examples of the voltage-current characteristics of the $\mathrm{YBa}_{2} \mathrm{Cu}_{3} \mathrm{O}_{7-\delta} / \mathrm{Ag}$ (black points) and $\mathrm{Nd}_{1,75} \mathrm{Ce}_{0,15} \mathrm{CuO} \mathrm{O}_{4-y} / \mathrm{Ag}$ ( red points) heterocontacts with resistive switching. $T=300 \mathrm{~K}$. Upper left corner - heterocontact scheme; Lower right corner - temperature dependences of $\mathrm{YBa}_{2} \mathrm{Cu}_{3} \mathrm{O}_{7-\delta} / \mathrm{Ag}$ heterojunction resistance in Off and On states.

to disorder only. At the same time the BRS effect was considerably stronger in the $\mathrm{YBCO} / \mathrm{Ag}$ heterocontacts, and the switching pattern corresponded to the hole-doped systems (Figure 2). As it is seen from inset in the Figure, superconducting transition is observed in the On state. It means that the electric field has induced oxygen diffusion into the controlled region and the Tc value is close to optimal one for $\mathrm{YBCO}$.

\section{Conclusions}

- The bipolar resistive switching has been experimentally observed in NCCO- and YBCO-based film heterojunctions.

- It has been shown that the surface layer of the studied HTSC films is oxygen degraded and can serve as an interface in HTSC perovskite-based heterostructures to create memory elements based on resistive switching.

- The switching polarity and the BRS effect value in the electron- and hole-doped compounds reflect the pattern of their oxygen doping.

\section{Competing interests}

The authors declare that they have no competing interests.

\section{Authors' contributions}

NAT carried out out the bipolar resistive switching effect measurements and drafted the manuscript. IYB carried out bipolar resistive switching effect measurements and discussed results. AAl produced thin HTSC films by pulsed laser deposition, drafted the manuscript. AMI carried out photoelectron spectroscopy measurements and ion milling, discussed results. IMS carried out XRD measurements and discussed results. All authors read and approved the final manuscript.

\section{Acknowledgements}

The work was financially supported by the Program of the Division of Physical Sciences RAS "Physics of Novel Materials and Structures", the program of the Presidium RAS "Quantum Condensed Matter Physics" and the Russian Foundation for Basic Research (project 11-02-01174a).

\section{Author details}

${ }^{1}$ Institute of Solid State Physics RAS, Chernogolovka, Russia. ${ }^{2}$ Institute of Microelectronics Technology and High Purity Materials RAS, Chernogolovka, Russia. ${ }^{3}$ National Research Nuclear University "MEPhl", Moscow, Russia.

Received: 27 June 2013 Accepted: 10 August 2013

Published: 15 August 2013

\section{References}

Dagotto E (1994) Correlated electrons in high-temperature superconductors. Rev Mod Phys 66: 763-840

Gauthier J, Gagné S, Renaud J, Gosselin ME, Fournier P, Richard P (2007) Different roles of cerium substitution and oxygen reduction in transport in $\mathrm{Pr}_{2-x} \mathrm{Ce}_{x} \mathrm{CuO}_{4}$ thin films. Phys. Rev. B 75: 024424

Higgins JS, Dagan Y, Barr MC, Weaver BD, Greene RL (2006) Role of oxygen in the electron-doped superconducting cuprates. Phys Rev B 73: 104510

Inoue IH, Yasuda S, Akinaga H, Takagi H (2008) Nonpolar resistance switching of metal/binary-transition-metal oxides/metal sandwiches:

homogeneous/inhomogeneous transition of current distribution. Phys Rev B 77: 035105

Jiang W, Mao SN, Xi XX, Jiang X, Peng JL, Venkatesan T, Lobb CJ, Greene RL (1994) Anomalous Transport Properties in Superconducting $\mathrm{Nd}_{185} \mathrm{Ce}_{015} \mathrm{CuO}_{4-\delta}$. Phys Rev Lett 73(9): 1291-1294

Jorgensen JD, Veal BW, Paulikas AP, Nowicki LJ, Crabtree GW, Claus H, Kwok WK (1990) Structural properties of oxygen-deficient $\mathrm{YBa}_{2} \mathrm{Cu}_{3} \mathrm{O}_{7-\delta}$. Phys Rev B 41: 1863-1877

Kang HJ, Dai P, Campbell BJ, Chupas PJ, Rozenkranz S, Lee PL, Huang Q, Li S, Komiya S, Ando Y (2007) Microscopic annealing process and its impact on superconductivity in T'-structure electron-doped copper oxides. Nat Mater 6: 224-229

Mao SN, Jiang W, Xi XX, Li Q, Peng JL, Greene RL, Venkatesan T, Beesabathina DP, Salamanca-Riba L, Wu XD (1995) Characteristics of oxygen over-reduced $\mathrm{Nd}_{1.85} \mathrm{Ce}_{0.15} \mathrm{CuO}_{4-y}$ films. Appl Phys Lett 66(16): 2137-2139 
Mang PK, Larochelle S, Mehta A, Vajk OP, Erickson AS, Lu L, Buyers WJL, Marshall AF, Prokes K, Greven M (2004) Phase decomposition and chemical inhomogeneity in $\mathrm{Nd}_{2-x} \mathrm{Ce}_{x} \mathrm{CuO}_{4 \pm \delta}$. Phys Rev B 70: 094507

Meijer GI (2008) Who wins the nonvolatile memory race? Science 319: $1625-1626$

Orenstein J, Wishwanath A (2010) Breaking through to the other side. Nat Phys 6: $566-567$

Sawa A (2008) Resistive switching in transition metal oxides. Mater Today 11(6): 28-36

Tulina NA (2007) Colossal electroresistance and electron instability in strongly correlated electron systems. Phys-Uspekhi 50(11): 1171-1178

Tulina N, Borisenko I, Ivanov A, Ionov A, Shmytko I, Menushenkov A (2011) Resistive switching effect in thin-film heterojunctions based on electron-doped $\mathrm{Nd}_{2-x} \mathrm{Ce}_{x} \mathrm{CuO}_{4-y}$ superconductor. Bull Russ Acad Sci : Physics 75(5): 605-608

Tulina NA, lonov AM, Chaika AN (2001) Reversible electrical switching at the $\mathrm{Bi}_{2} \mathrm{Sr}_{2} \mathrm{CaCu}_{2} \mathrm{O}_{8+y}$ surface in the normal metal $\mathrm{Bi}_{2} \mathrm{Sr}_{2} \mathrm{CaCu}_{2} \mathrm{O}_{8+y}$ single crystal heterojunction. Physica C 366: 23-30

Tulina NA, Klinkova (2007) Reversal of the resistive switching effect in electron-doped $\mathrm{Ba}_{0.6} \mathrm{~K}_{0.4} \mathrm{BiO}_{3-x}$. JETP 105: 238-240

Tulina NA, Sirotkin W (2004) Electron instability in doped-manganites-based heterojunctions. Physica C 400: 105-110

Waser R, Aono M (2007) Nanoionics-based resistive switching memories. Nat Mat 6: 833-840

Yang JJ, Pickett MD, Li X, Ohlberg DAA, Stewart DR, Williams S (2008) Memristive switching mechanism for metal/oxide/metal nanodevices. Nat Nanotechnol 3: 429-433

doi:10.1186/2193-1801-2-384

Cite this article as: Tulina et al: Oxygen doping of HTSC and resistive switching in HTSC-based heterostructures. SpringerPlus 2013 2:384.

\section{Submit your manuscript to a SpringerOpen ${ }^{\circ}$ journal and benefit from:}

- Convenient online submission

- Rigorous peer review

- Immediate publication on acceptance

- Open access: articles freely available online

- High visibility within the field

- Retaining the copyright to your article

Submit your next manuscript at $\gg$ springeropen.com 